\title{
Temperature-Regulated IncX3 Plasmid Characteristics and the Role of Plasmid-Encoded H-NS in Thermoregulation
}

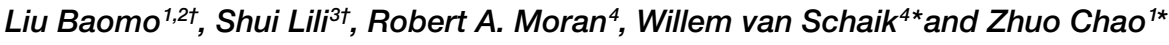 \\ 'State Key Laboratory of Respiratory Disease, The First Affiliated Hospital of Guangzhou Medical University, Guangzhou, China, \\ ${ }^{2}$ Department of Respiratory and Critical Care Medicine, The First Affiliated Hospital of Sun Yat-sen University, Guangzhou, \\ China, ${ }^{3}$ Department of Pulmonary and Critical Care Medicine, The First Affiliated Hospital of Chongqing Medical University, \\ Chongqing, China, ${ }^{4}$ Institute of Microbiology and Infection, University of Birmingham, Birmingham, United Kingdom
}

OPEN ACCESS

Edited by:

Fenni Zhang,

Zhejiang University, China

Reviewed by:

Yukihiro Akeda,

Osaka University, Japan

Susu He,

Nanjing University, China

*Correspondence:

Willem van Schaik

W.vanSchaik@bham.ac.uk

Zhuo Chao

chao_sheep@263.net

tThese authors have contributed

equally to this work

Specialty section:

This article was submitted to

Antimicrobials, Resistance and

Chemotherapy,

a section of the journal

Frontiers in Microbiology

Received: 27 August 2021

Accepted: 17 November 2021

Published: 06 January 2022

Citation:

Baomo L, Lili S, Moran RA, van Schaik W and Chao Z (2022)

Temperature-Regulated IncX3

Plasmid Characteristics and the Role

of Plasmid-Encoded H-NS in

Thermoregulation.

Front. Microbiol. 12:765492.

doi: 10.3389/fmicb.2021.765492
Carbapenem-resistant Enterobacteriaceae (CRE) are a critical public health problem worldwide. Globally, IncX3-type plasmids have emerged as the predominant vehicles carrying the metallo- $\beta$-lactamase gene b/a $a_{\text {NDM }}$. Although $b / a_{\text {NDM }}$-bearing IncX3 plasmids have been found in various hosts from diverse environments, whether their transfer and persistence properties vary under different conditions and what factors influence any variation is unknown. By observing the effects of different temperatures on IncX3 plasmid conjugation rates, stability, and effects on host fitness in Escherichia coli, we demonstrate that temperature is an important determinant of plasmid phenotypes. The IncX3 plasmid pGZIncX3 transferred at highest frequencies, was most stable and imposed lower fitness costs at $37^{\circ} \mathrm{C}$. Temperature-regulated variation in pGZIncX3 properties involved a thermoregulated plasmid-encoded H-NS-like protein, which was produced at higher levels at $30^{\circ} \mathrm{C}$ and $42^{\circ} \mathrm{C}$ and inhibited the expression of type IV secretion system genes involved in conjugation. These findings suggest that bla $_{\mathrm{ND}}$-bearing IncX3 plasmids are adapted to carriage by enterobacteria that colonize mammalian hosts and could explain the rapid dissemination of these plasmids among human-associated species, particularly in hospital settings.

Keywords: IncX3, carbapenem resistance Enterobacteriaceae, temperature, plasmid-encoded hns, fitness

\section{INTRODUCTION}

Carbapenem-resistant Enterobacteriaceae (CRE) are a critical public health problem globally, as they can exhibit resistance to almost all available antibiotics and are responsible for extensive morbidity and mortality (Logan and Weinstein, 2017). Bacteremia due to CRE can result in mortality rates higher than $40 \%$ in China (Zhang et al., 2018). New Delhi metallo- $\beta$-lactamase (NDM)-type carbapenemase is the major mechanism mediating carbapenem resistance in approximately $40 \%$ of CRE isolates (Bush and Bradford, 2020). Although the bla $a_{\mathrm{NDM}}$ gene can be found inserted in the chromosome, the substantial majority of carriage is linked to plasmids, which contribute significantly to the rapid dissemination of carbapenem resistance (Khan et al., 2017). 
IncX3 plasmids are emerging as the predominant plasmid type carrying $b l a_{\mathrm{NDM}}$ worldwide, with approximately one third of $b l a_{\mathrm{NDM}}$-carrying plasmids deposited in GenBank containing IncX3-type replicons (Ma et al., 2020). Additionally, IncX3 plasmids appear to function as a platform for the evolution of NDM enzymes, as diverse $b{ } a_{\mathrm{NDM}}$ gene variants have been found in plasmids of the same replicon type (Ma et al., 2020). Strains containing $b l a_{\mathrm{NDM}}$-bearing IncX3 plasmids have been isolated from clinical settings (Zhou et al., 2020), companion animals (Nigg et al., 2019), agricultural settings (Zhang et al., 2019), and the environment (Zhai et al., 2020), which suggests they can persist in their hosts under various conditions, including different temperatures. The mechanisms that facilitate IncX3 plasmid persistence and dissemination at different temperatures are unknown.

In Escherichia coli and other Gram-negative bacteria, the histone-like nucleoid structuring (H-NS) protein has been shown to take part in the regulation of chromosomal transcriptional networks (Dorman and Ni Bhriain, 2020). Many genes encoding different H-NS family proteins have been found in microbial chromosomes as well as in plasmids (Shintani et al., 2015). Our previous work revealed that a H-NS protein homolog encoded by an IncX3-plasmid inhibits plasmid transfer and partitioning (Liu et al., 2020). Plasmid-encoded H-NS-like proteins are also known to play an important role in the regulation of transfer genes in IncH plasmids. The well-studied IncHI1 plasmid R27 exhibits thermosensitive variation in conjugative transfer frequencies modulated by host- and plasmidencoded H-NS-like proteins (Forns et al., 2005).

In this study, we examined the IncX3 plasmid-encoded H-NS-like protein and characterized its effect on plasmid phenotypes at various temperatures. We show that this protein is essential in linking plasmid phenotypes with temperatures.

\section{MATERIALS AND METHODS}

\section{Bacterial Strains, Plasmids, and Culture Conditions}

Bacterial strains and plasmids presented in this study are detailed in Table $\mathbf{1}$.

Strains J330(phns), J330(pACYC184), Pkhns(phns), and Pkhns(pACYC184) were obtained by transforming the corresponding plasmids into these strains by electrotransformation. Bacteria were stored in $25 \%$ glycerol medium at $-80^{\circ} \mathrm{C}$ and recovered and cultured in Luria broth (LB) medium (containing $10 \mathrm{~g} \mathrm{NaCl}, 10 \mathrm{~g}$ tryptone, and $5 \mathrm{~g}$ yeast per liter) at $37^{\circ} \mathrm{C}$ unless otherwise mentioned.

\section{Conjugation Experiments}

Conjugation assays with pGZIncX3 and its derivatives were conducted as previously reported (Forns et al., 2005; Wang et al., 2017), using rifampin-resistant E. coli Ec600 as a recipient strain. Briefly, cultures of donor and recipient strains were grown overnight with shaking at different temperatures $\left(25,30,37\right.$, or $\left.42^{\circ} \mathrm{C}\right)$ in LB medium. The mating mixture, with a 1:2 donor to recipient ratio, was incubated at the
TABLE 1 | All test strains in this work.

\begin{tabular}{|c|c|c|}
\hline Strains & Description & Reference \\
\hline J53 & E. coli $\mathrm{F}^{-}$met pro Azir & $\begin{array}{l}\text { Matsumura et al., } \\
2018\end{array}$ \\
\hline J330 & $\begin{array}{l}\text { J53 with pGZIncX3 } \\
\text { transferred naturally }\end{array}$ & Liu et al., 2020 \\
\hline J330(phns) & $\begin{array}{l}\text { J53 with pACYC184phns } \\
\text { transferred by } \\
\text { electroporation }\end{array}$ & This work \\
\hline J330(pACYC184) & $\begin{array}{l}\text { J53 with pACYC184 } \\
\text { transferred by } \\
\text { electroporation }\end{array}$ & This work \\
\hline Pkhns & $\begin{array}{l}\text { J53 with pGZIncX3 } 3 \text { phns } \\
\text { transferred by } \\
\text { electroporation }\end{array}$ & Liu et al., 2020 \\
\hline Pkhns(phns) & $\begin{array}{l}\text { J330 with pACYC184 } \\
\text { phns transferred by } \\
\text { electroporation }\end{array}$ & This work \\
\hline Pkhns(pACYC184) & $\begin{array}{l}\text { J330 with pACYC184 } \\
\text { transferred by } \\
\text { electroporation }\end{array}$ & This work \\
\hline Ec600 & $\begin{array}{l}\text { E.coli recipient strains for } \\
\text { conjugation experiments }\end{array}$ & Laboratory stock \\
\hline \multicolumn{3}{|l|}{ Plasmids } \\
\hline pGZIncX3 & $\begin{array}{l}\text { blaNDM }{ }^{+} \text {, IncX3, sharing } \\
99 \% \text { similarity with } \\
\text { pNDM-HN380 }\end{array}$ & Liu et al., 2020 \\
\hline pGZIncX3sphns & $\begin{array}{l}\text { pGZIncX3 knocking } \\
\text { down plasmid-encoded } \\
\text { phns gene }\end{array}$ & Liu et al., 2020 \\
\hline pACYC184 & $\begin{array}{l}\text { Chl', Tetr, blank vector for } \\
\text { gene complementary } \\
\text { hns::Chl', pACYC184 }\end{array}$ & Rose, 1988 \\
\hline pACYC184phns & $\begin{array}{l}\text { with inserted IncX3 } \\
\text { plasmid-encoded phns } \\
\text { gene }\end{array}$ & This work \\
\hline
\end{tabular}

corresponding temperature, without shaking overnight and then plated in LB agar supplemented with rifampin $\left(200 \mu \mathrm{g} \mathrm{ml}^{-1}\right)$ single (for recipients) or with meropenem $\left(2 \mu \mathrm{g} \mathrm{ml}^{-1}\right.$; for transconjugants). The plates were incubated at $37^{\circ} \mathrm{C}$, and the mating frequency was calculated as the number of transconjugants per recipient.

\section{Plasmid Stability Test}

Plasmid stability was determined as previously reported with minor modifications (Gao et al., 2020). Briefly, cultures were incubated at $25,30,37$, or $42^{\circ} \mathrm{C}$ in a shaking water bath $(200 \mathrm{rpm})$ and were diluted 1,000-fold in antibiotic-free LB broth. After $24 \mathrm{~h}$, cultures were serially diluted and plated on antibiotic-free LB agar and LB agar containing meropenem $\left(0.5 \mu \mathrm{g} \mathrm{ml}^{-1} \mathrm{~L}\right)$. The retention rate of the $b l a_{\mathrm{NDM}-1}$ gene was calculated by dividing the number of colonies that grew on meropenem-containing LB agar by the number of colonies on antibiotic-free LB agar. In addition, 10 colonies were randomly selected and subjected to PCR validation of the $b l a_{\mathrm{NDM}-1}$ gene. [It is unlikely that the chromosomal integration of NDM gene in plasmid stability test occurred. In our previous published work for IncX3 plasmid (Liu et al., 2020), we have proved 


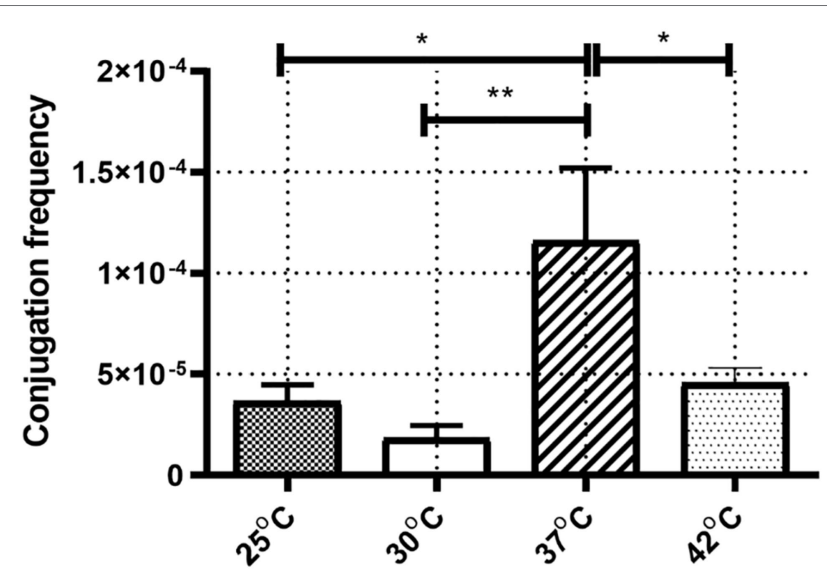

FIGURE 1 | The conjugation frequency of IncX3 at four corresponding temperature. The conjugation frequency was calculated as transconjugants per recipient. Error bars indicate the standard deviations for six triplicate samples. ${ }^{*} p<0.05 /{ }^{* \star} p<0.01$ versus the result for conjugation frequency at $37^{\circ} \mathrm{C}$

that "PCR tests revealed that the blaNDM-1 gene was maintained by transconjugants and wild-type isolates for 1,000 generations. In addition, the location of the blaNDM-1 gene did not change in the decedents, as shown by Southern blotting." The $24 \mathrm{~h}$ passage in this plasmid stability test could not lead to NDM-1 chromosomal integration].

\section{Competition Experiments}

To assess the cost of carriage of $b l a_{\mathrm{NDM}^{-}}$-bearing plasmid pGZIncX3 in $E$. coli at various temperatures $(25,30,37$, or $42^{\circ} \mathrm{C}$ ), growth competition experiments between E. coli J53 and pGZIncX3-carrying derivatives were performed in LB broth using a previously published protocol (Johnson et al., 2015). Briefly, colonies from each strain were initially grown individually in $\mathrm{LB}$ broth at $25,30,37$, or $42^{\circ} \mathrm{C}$ overnight with shaking. On day 0 , bacteria were pelleted and suspended in equal volumes of PBS, and then inoculated at a volume of $25 \mu \mathrm{l}$ each for competing strains into $5 \mathrm{ml}$ of sterile LB broth. Competitions were performed for $24 \mathrm{~h}$ at each temperature, after which the cultures were serially diluted and plated on LB agar without antibiotic for total bacterial counts and on LB agar containing meropenem $\left(0.5 \mu \mathrm{g} \mathrm{ml}^{-1}\right)$ to select for cells containing pGZIncX3. All competition experiments were performed a minimum of three times. Fitness cost was calculated as follows: $\log 2(a / b) / \log 2(c / d)$, where $a=$ the number of plasmidfree cells at $24 \mathrm{~h}, b=$ the number of plasmid-free cells at $0 \mathrm{~h}$, $c=$ the number of plasmid-containing cells at $24 \mathrm{~h}$, and $d=$ the number of plasmid-containing cells at $0 \mathrm{~h}$ (Johnson et al., 2015).

\section{Plasmid Construction}

For performing complementation experiments, the plasmidencoded phns gene (positions 28,899-29,354 in GenBank accession JX104760) of IncX3 plasmid pNDM-HN380 (JX104760) was cloned into the pACYC184 vector. After amplifying the phns gene using PrimeSTAR ${ }^{\circledR}$ HS DNA Polymerase (Takara), the PCR fragment was purified using TaKaRa MiniBEST DNA Fragment Purification Kit (Takara) and digested with EcoRI and BamHI restriction enzymes (Takara). Ligation was performed in pACYC184 digested with the same restriction enzymes. The resulting plasmid (pACYC184phns) was transformed into $E$. coli $\mathrm{DH} 5 \alpha$ and picked in the presence of chloramphenicol. Primers were used for sequencing to confirm correct in-frame insertion of the phns gene.

\section{Real-Time Polymerase Chain Reaction}

RNA of all samples was isolated using the E.Z.N.A. Bacterial RNA Kit (Omega Bio-tek). The qPCR was conducted with primers targeting the chromosome-encoded idnT (reference gene) and the plasmid gene as below. The isolated RNA was then transcribed into cDNA using a PrimeScript ${ }^{\mathrm{TM}}$ RT Master Mix (TaKaRa). Real-time polymerase chain reaction (RT-PCR) was used to quantify the expression of genes related to conjugation (pilX4, pliX5, pilX9, and pilX10). RT-PCR was conducted using the TB Green ${ }^{\mathrm{TM}}$ Premix Ex Taq ${ }^{\mathrm{TM}}$ II (TaKaRa) with the standard procedure for two-step PCR amplification (LightCycler96, Roche, Switzerland).

\section{Data Analysis}

GraphPad Prism 8.0 was used for statistics analysis and figure drawing. ANOVA was used to analyze the difference between groups.

\section{RESULTS}

\section{The Transfer of the IncX3 Plasmid pGZIncX3 to $E$. coli Is Regulated by Temperature}

The frequencies of IncX3 plasmid pGZIncX3 conjugative transfer from J330 to E. coli Ec600 were determined at different temperatures (Figure 1). ANOVA analysis showed a significant difference between conjugation frequencies at different temperatures $(p=0.002)$. Conjugation frequency was highest at $37^{\circ} \mathrm{C}\left(1.2 \times 10^{-4}\right)$ while incubation at $25^{\circ} \mathrm{C}, 30^{\circ} \mathrm{C}$, and $42^{\circ} \mathrm{C}$ reduced transfer rates $\left(3.7 \times 10^{-5}, 1.8 \times 10^{-5}\right.$, and $4.6 \times 10^{-5}$, respectively) with the lowest conjugation frequencies at $25^{\circ} \mathrm{C}$ or $30^{\circ} \mathrm{C}$. There was no significant difference between conjugation frequency at $25^{\circ} \mathrm{C}$ and $30^{\circ} \mathrm{C}$.

\section{H-NS Gene Expression Varied With Differences in Temperature}

To study the mechanism of the temperature dependence of plasmid conjugation, stability, and fitness, we focused on the pGZIncX3 gene phns, which encodes a H-NS-like protein. We previously showed that this gene is an inhibitor of plasmid transfer and partitioning (Liu et al., 2020). To further characterize the role of phns, we first determined the expression of phns under different temperatures, which revealed that phns was more highly expressed at $30^{\circ} \mathrm{C}$ and $42^{\circ} \mathrm{C}$ that at $25^{\circ} \mathrm{C}$ and $37^{\circ} \mathrm{C}$ (Figure 2). To avoid the impact of plasmid copy numbers 
(PCN) at different degree on gene expression, we compared them and found no difference (see Supplementary data 1, Figure n1). Moreover, phns itself did not affect PCN (Supplementary data 1, Figure n2).

\section{Plasmid-Encoded H-NS Affects Thermoregulation of IncX3 Transfer}

To confirm the role of phns gene in plasmid fitness, we obtained pGZIncX3 derivatives harboring a phns deletion and the complemented mutant. E. coli strain J53 harboring either wildtype (WT) pGZIncX3 or its phns deleted or complemented derivatives were used as the donor in mating assays performed at four different temperatures (Figure 3).

At $37^{\circ} \mathrm{C}$, conjugation results showed that phns deletion derepressed plasmid transfer. Lack of phns gene promoted the transfer rates of pGZIncX3 with the phns deletion (Pkhns) to Ec600 by 4.2 -fold. Complementation of phns gene restored the transfer rates of Pkhns. We also found a reduction of conjugation frequencies in $\mathrm{J} 330$ complemented with phns.

At 25,30 , and $42^{\circ} \mathrm{C}$, the plasmid-encoded $\mathrm{H}$-NS-like protein also exerted a negative impact on conjugation. At every temperature, J330 with phns complementation exhibited decreased transfer rates relative to the parental strain J330 while phnsnull strains showed robust transfer ability with high conjugation rates.

\section{Temperature Regulates the Conjugative Transfer of IncX3 Plasmid by Regulating the Expression of H-NS-Like Protein}

In order to verify that temperature affects the conjugation frequency of plasmids by regulating the expression of the phns gene, we compared the conjugation frequency of pGZIncX3

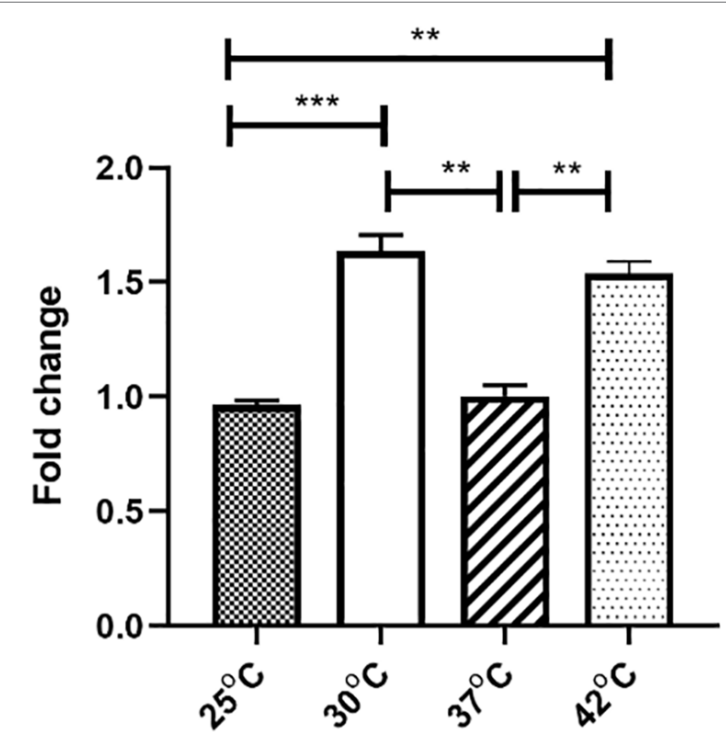

FIGURE 2 | The effect of four temperatures on pGZIncX3 plasmid gene phns expression levels. Variances between different temperature groups were shown with ${ }^{\star}(p<0.05),{ }^{\star *}(p<0.01)$, and ${ }^{\star * \star}(p<0.001)$. and pGZIncX3 $\Delta$ hns to the recipient strain EC600 at different temperatures by using a plasmid conjugation experiment. As shown in Figure $\mathbf{4 A}$, when the phns gene is deleted, there is no difference in the conjugation frequency of plasmid pGZIncX3 3 hns at 30 degrees, 37 degrees, and 42 degrees. Figure $4 \mathrm{~B}$ shows the comparison of the conjugation frequency of pGZIncX3 in J330 at 30 degrees, 37 degrees, and 42 degrees with the conjugation frequency of pGZIncX3 in the phns gene overexpression strain $\mathrm{J} 330$ (phns) at 37 degrees. The frequency is significantly reduced at 30 degrees and 42 degrees, which is consistent with the decrease in the conjugation frequency of pGZIncX3 when the phns gene is overexpressed at 37 degrees, suggesting that phns gene is upregulated at 30 degrees and 42 degrees, further inhibiting plasmid conjugative transfer.

\section{Transcription of Several Genes Encoded in pGZIncX3 Is Enhanced in a phns Deletion Mutant}

We used RT-PCR to test if the plasmid-encoded H-NS-like protein has an impact on the transcription of plasmid genes involved in conjugation. In our previous work (Liu et al., 2020), we have analyzed the transcriptome differences between J330 and Pkhns, which showed that the expressions of all genes from pilX operon (including pilX1,2,3,4,5,6,8,9,10,11) are elevated in Pkhns. These genes encode constituents of a type IV secretion system (T4SS) and have been shown to be involved in conjugation (Chen et al., 2009). The pilX4, pilX5, pilX9, and pilX10 genes were chosen to represent expression of the pilX operon at different temperatures (Figure 5).

We found that the phns gene reduces the expression of conjugation genes at various temperature which is corresponding to the results of conjugation test above. At low temperature $\left(25^{\circ} \mathrm{C}\right.$ or $\left.30^{\circ} \mathrm{C}\right)$, the expression of pilX4,5,9,10 in Pkhns could be more than 50 -fold upregulated compared with J330. This experiment demonstrated that although low temperature could inhibit conjugation and the expression of genes involved in conjugation, the deletion of the plasmid gene encoding the H-NS homolog could abolish the impact of temperature.

\section{The Impact of Temperature on Plasmid- Mediated Bacterial Fitness and Plasmid Stability in E. coli J53}

To explore the impact of temperature on plasmid stability and E. coli host fitness, pGZIncX3 was transferred into E. coli J53 by conjugation to create J330. The stability of pGZIncX3 in J330 was evaluated after growth in antibiotic-free medium (Figure 6). pGZIncX3 was most stable at $37^{\circ} \mathrm{C}$, with plasmid retention rate of $6.0 \%$, with significantly lower plasmid retention at $25^{\circ} \mathrm{C}, 30^{\circ} \mathrm{C}$, and $42^{\circ} \mathrm{C}$ (Figure 6A).

To evaluate the impact of pGZIncX3 on J330 fitness at different temperatures, growth competition experiments between J330 and plasmid-free J53 were performed at different temperatures. pGZIncX 3 had a higher fitness cost at $30^{\circ} \mathrm{C}$ and $42^{\circ} \mathrm{C}$ than at $37^{\circ} \mathrm{C}$ (Figure 6B), but no significant difference in fitness cost was observed between $25^{\circ} \mathrm{C}$ and $37^{\circ} \mathrm{C}$. 


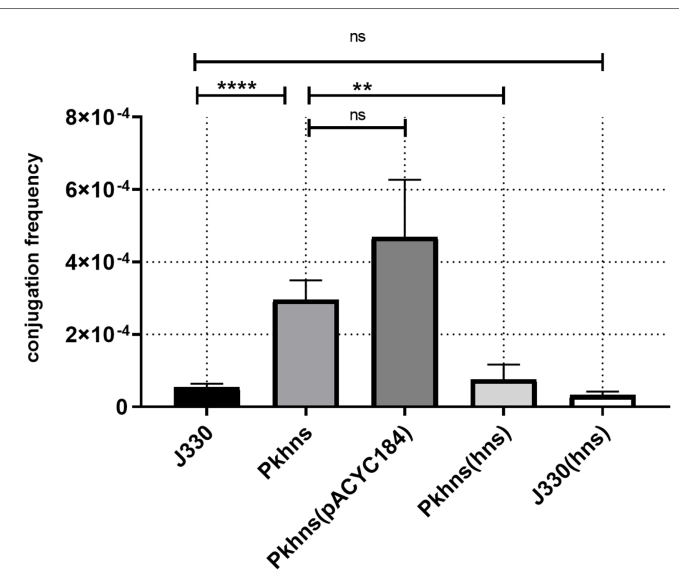

$25^{\circ} \mathrm{C}$

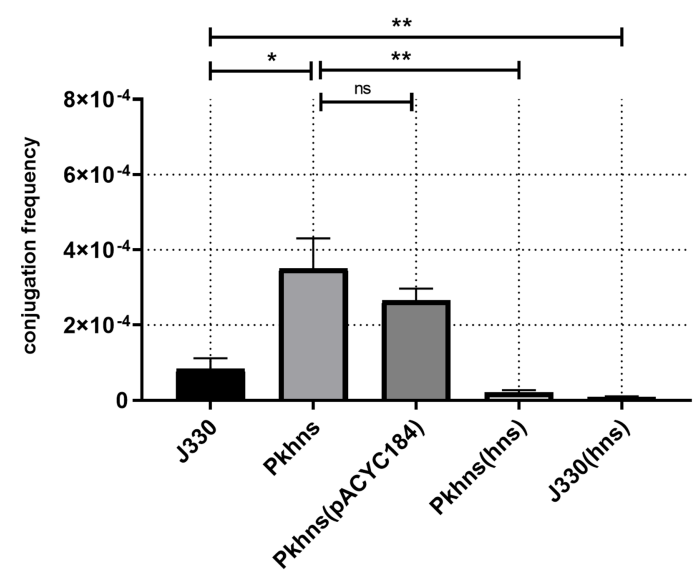

$37^{\circ} \mathrm{C}$
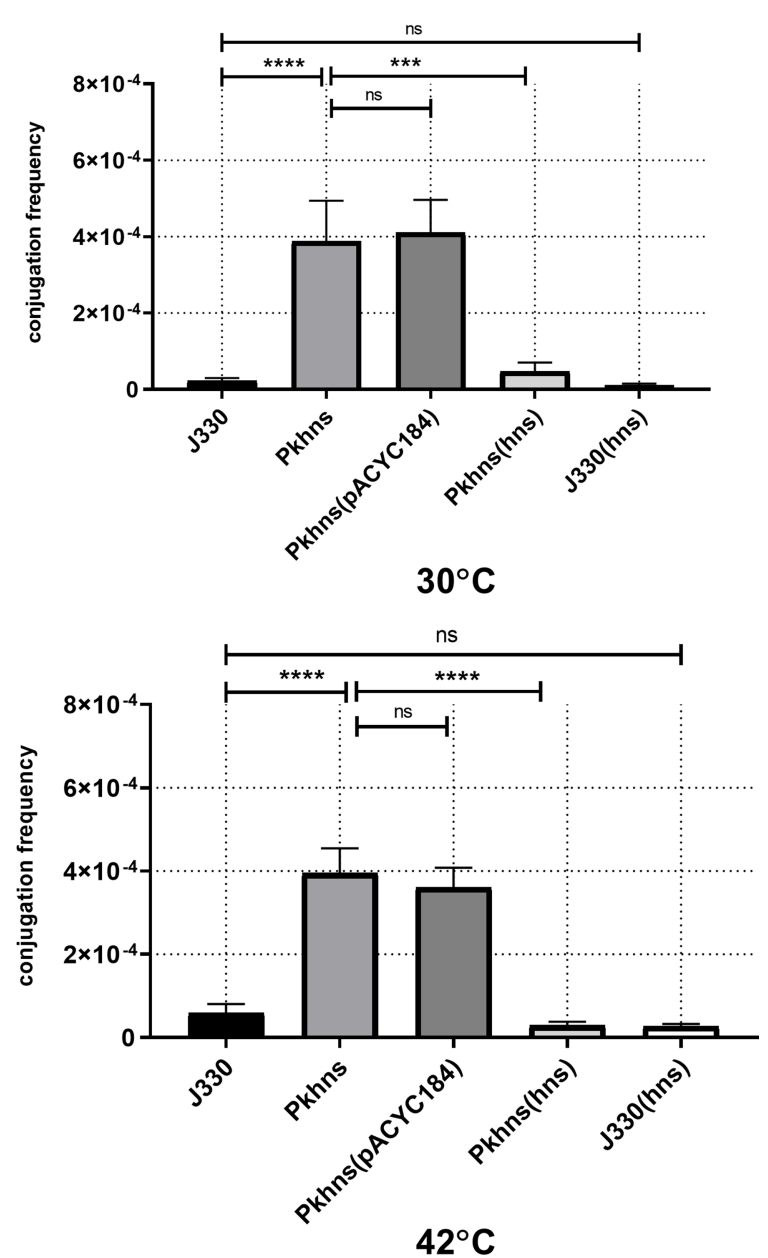

FIGURE 3 | Plasmid-encoded H-NS-like protein represses plasmid transfer at different temperatures. Conjugation frequency at various temperature was evaluated for J330, Pkhns(phns-null strains), corresponding phns complementary strains Pkhns(hns), J330(hns), and corresponding blank-vector complementary strains J330(pACYC184), Pkhns(pACYC184). Variances between different temperature groups were shown with * $p<0.05)$, ${ }^{\star *}(p<0.01)$, and ${ }^{\star \star *}(p<0.001)$,

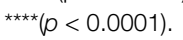

\section{The Impact of phns on Plasmid Stability and Host Fitness Cost at Various Temperature}

To test if plasmid stability also changed through regulation of the plasmid-encoded H-NS protein, we compared stability of pGZIncX3 in the E. coli host at a range of temperature. Under all temperatures tested, phns-null J330 derivate strains showed higher plasmid retention rates versus wild-type strains (Figure 7A).

We also tested the effects of phns on bacterial fitness. Deletion of phns slightly increased the fitness of plasmid-containing E. coli fitness at 25 and $30^{\circ} \mathrm{C}$, whereas overall fitness at 37 and $42^{\circ} \mathrm{C}$ did not change significantly (Figure 7B).

\section{DISCUSSION}

The role of $b l a_{\mathrm{NDM}}$-bearing IncX3 plasmids in the spread of carbapenem resistance makes them an important threat to global public health. Our results show that the phenotypes conferred by the IncX3 plasmid pGZIncX3 are affected by environmental temperature and the plasmid-encoded H-NSlike protein is involved in this regulation. In this study, we focused on the effects of four distinct temperatures on IncX3 plasmid phenotypes as they are representative of environmental temperatures in many countries in Asia and the Middle East $\left(25^{\circ} \mathrm{C}\right.$ and $\left.30^{\circ} \mathrm{C}\right)$, the temperature of the human body $\left(37^{\circ} \mathrm{C}\right)$, and the body temperature of chickens $\left(42^{\circ} \mathrm{C}\right.$; Rozwandowicz et al., 2019).

Here, we demonstrate that the IncX3 plasmid pGZIncX3 could transfer at highest frequencies at $37^{\circ} \mathrm{C}$, suggesting that transfer of this important group of plasmids is highest in the guts of humans and other mammals, which is in line with previous work (Liakopoulos et al., 2018; Wang et al., 2018), that showed that the optimal temperature for conjugative transfer of IncX3 plasmids was $37^{\circ} \mathrm{C}$. In addition, we have shown that at $37^{\circ} \mathrm{C}$, pGZIncX3 was most stable in the absence of antibiotic selective pressure and imposed 
A

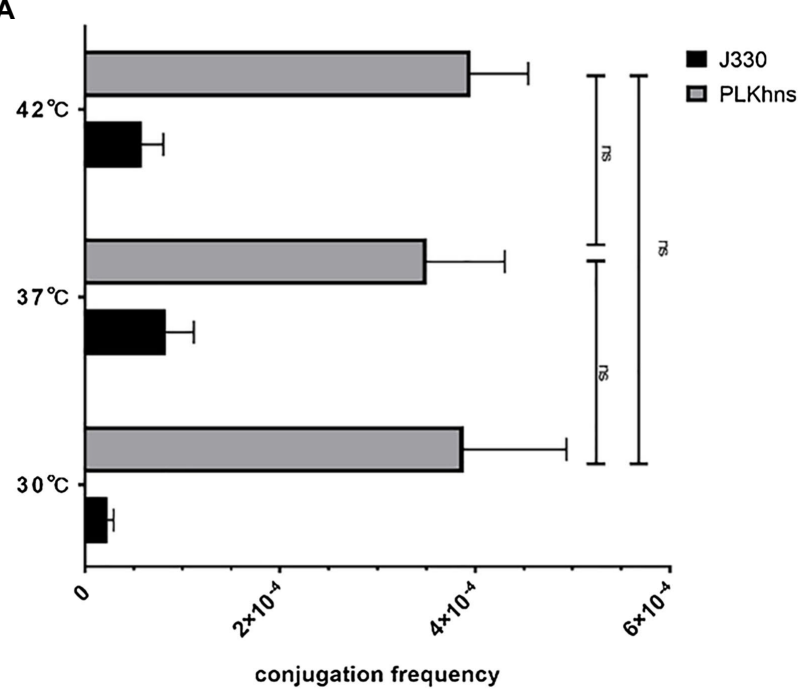

B

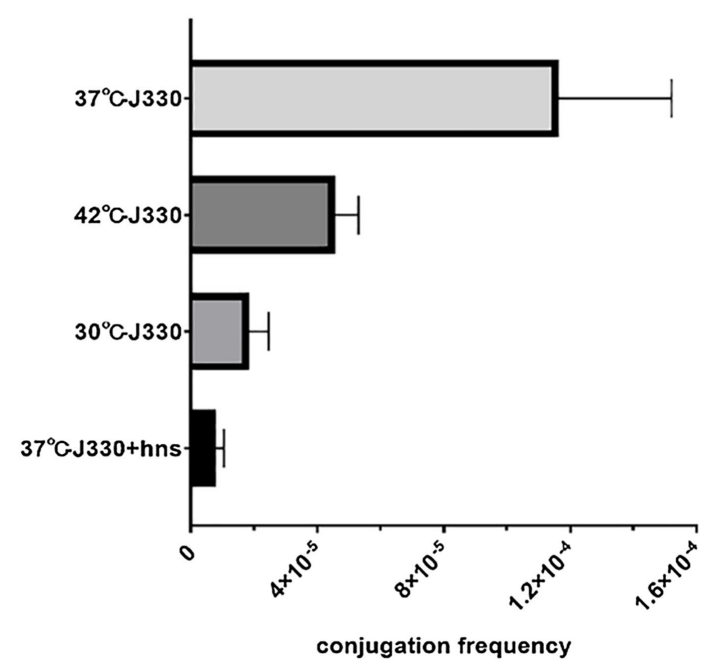

FIGURE 4 | (A) Comparison of the frequency of conjugative transfer between the phns gene deletion plasmid and the original plasmid at different temperatures. At different temperatures, the joint frequency of $p G Z I n c X 3 \Delta$ hns and pGZIncX3. (B) Comparison of conjugation frequency of $p G Z I n c X 3$ at three different temperatures and 37 degrees when phns gene was overexpressed.

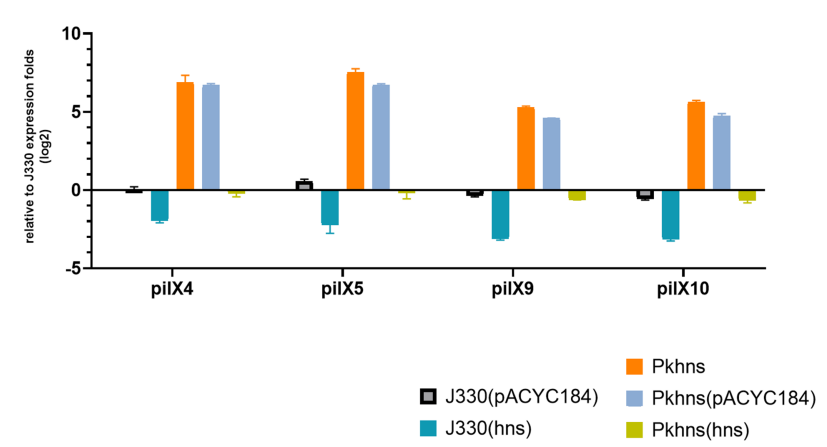

$25^{\circ} \mathrm{C}$

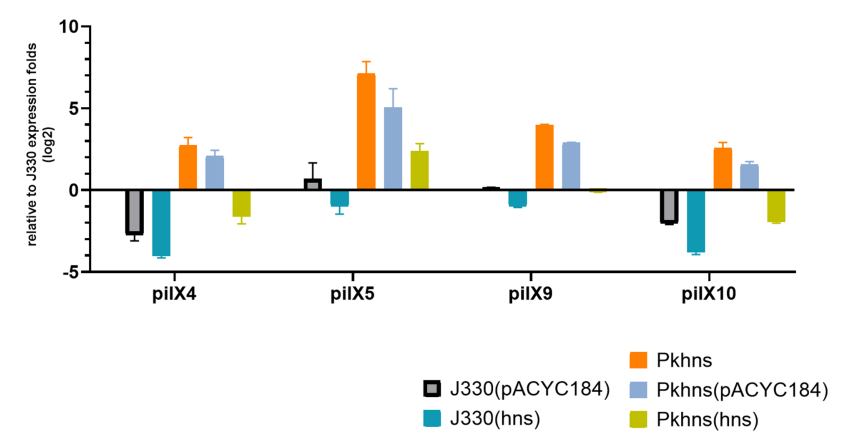

$37^{\circ} \mathrm{C}$

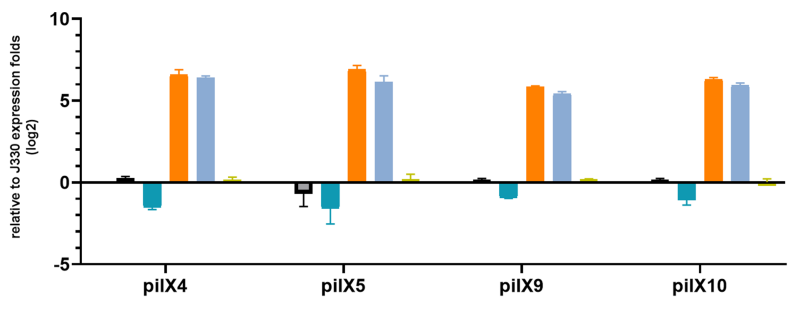

Pkhns

ㅁ J330(pACYC184) Pkhns(pACYC184)

J330(hns) Pkhns(hns)

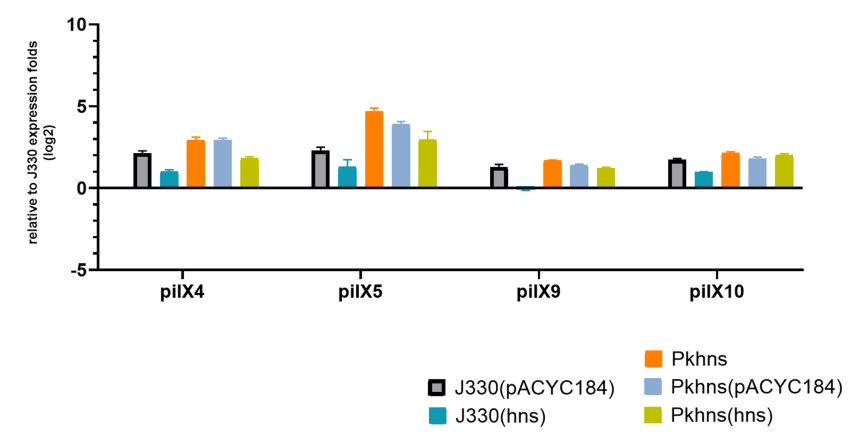

$42^{\circ} \mathrm{C}$

FIGURE 5 | At four different temperatures $\left(25,30,37\right.$, and $\left.42^{\circ} \mathrm{C}\right)$, the mRNA expression of T4SS-related gene pilX4, 5, 9, and 10 of six gene context-related strains: J330, Pkhns(phns-null strains), corresponding phns complementary strains Pkhns(hns), J330(hns), and corresponding blank-vector complementary strains J330(pACYC184), Pkhns(PACYC184). 

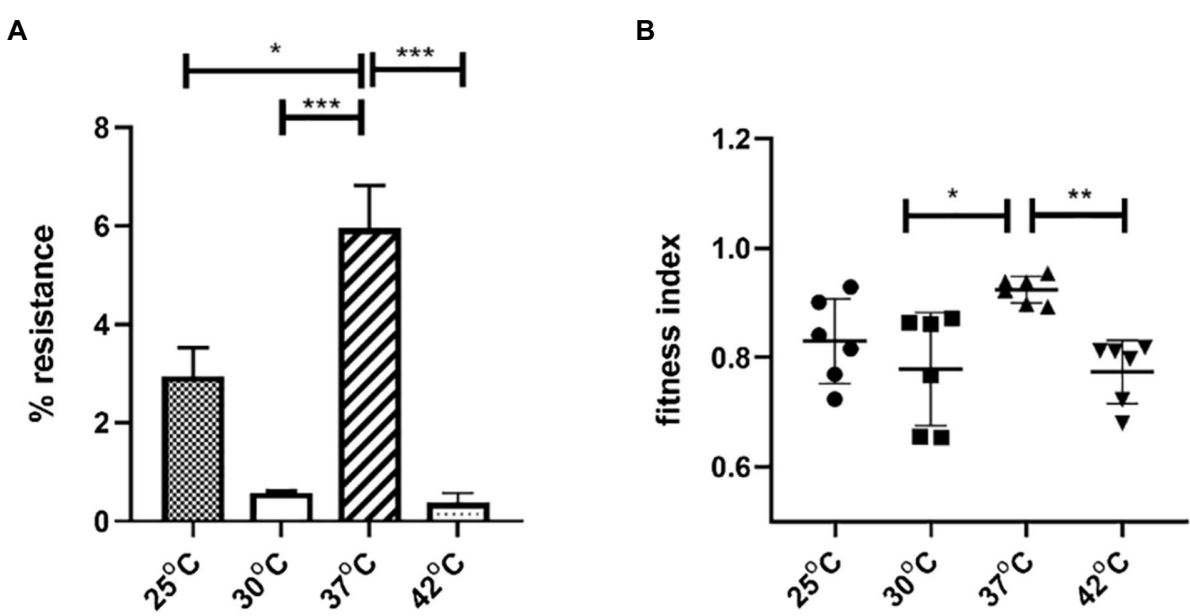

FIGURE 6 | The impact of temperatures on IncX3 plasmid stability (A) and fitness cost (B) in Escherichia coli. (A) Plasmid stability is estimated by the percent of plasmid-containing E. coli at $24 \mathrm{~h}$ as determined by resistance to meropenem. (B) Fitness index is calculated by formula described in Section "Materials and Methods." The higher fitness index represents better fitness of plasmid-containing strains. Significant differences between temperatures are indicated with asterisks: ${ }^{\star} p<0.05,{ }^{* \star} p<0.01$, and ${ }^{\star \star \star} p<0.001$.
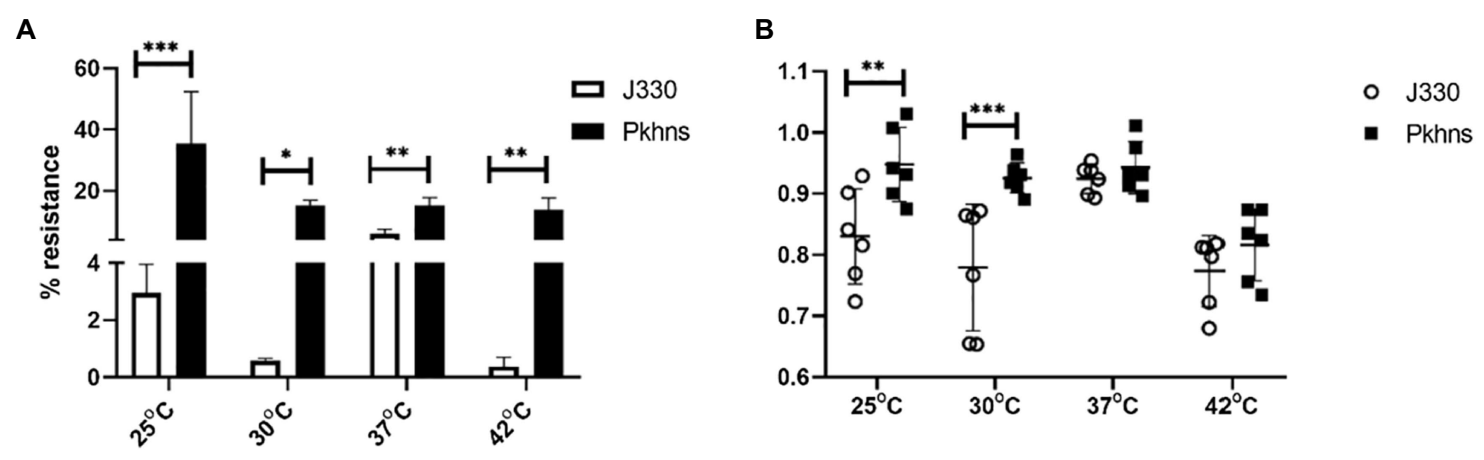

FIGURE 7 | At various temperature, the impact of plasmid-encoded H-NS-like protein on IncX3 plasmid stability (A) and fitness cost (B) in E. coli. (A) Plasmid stability is estimated by the percent of plasmid-containing $E$. coli at $24 \mathrm{~h}$ (\% resistance). (B) Fitness index is calculated by formula described in Section "Materials and Methods." The higher fitness index represents better fitness of plasmid-containing strains. Significant differences are indicated with * $(p<0.05),{ }^{* *}(p<0.01)$, and **** $(p<0.001)$.

the lowest fitness costs. It is not surprising that conjugation rates and stability properties are both optimized at $37^{\circ} \mathrm{C}$. As there is an evolutionary selection for small fitness effects of plasmids to ensure long-term persistence of the plasmids in the absence of selective pressures (Wein et al., 2019), these plasmids have adapted to life in gut commensals of mammals.

We found a new mechanism for IncX3 plasmid adaptation to different temperatures. We explored the potential mechanisms that link environmental temperature to plasmid stability, plasmid transfer, and fitness costs of pGZIncX3. Our study showed that growth at $30^{\circ} \mathrm{C}$ and $42^{\circ} \mathrm{C}$ caused the elevated expression level of the pGZIncX3 phns gene and we confirmed the role of this gene in suppressing conjugation in a temperature-dependent fashion. This phenomenon was linked to phns-dependent repression of the genes found in the pilX operon that encodes the IncX3 T4SS, namely, pilX4, pilX5, pilX9, and pilX10.

We also found that plasmid stability is repressed by phns under all temperatures tested. H-NS inhibition of conjugation leads to lower stability through the mechanisms mentioned above. Results in our study revealed the IncX3 plasmidencoded H-NS-like protein only slightly affects host fitness, in line with previous work on a different IncX3 plasmidencoded $h n s$-like gene (Ho et al., 2013). But, in previous work, the IncHIl plasmid-encoded Sfh protein, which is an H-NS homologue, was proposed to promote plasmid transfer to new bacterial hosts with little effects on bacterial fitness (Doyle et al., 2007). The discordant observations between these two plasmid-encoded H-NS homologs could 
be due to differences in host species (Salmonella spp. vs. E. coli), plasmid length (54 vs. $100 \mathrm{kbp}$ ), and host adaptive mutations (Takeda et al., 2011). Moreover, discordance of plasmid-encoded hns function between E. coli and Salmonella may be explained by significantly different hns-related transcription profiles as shown in the Doyle et al study (Doyle et al., 2007) and our previous work (Liu et al., 2020). The current consensus is that an increase in temperature (from $30^{\circ} \mathrm{C}$ to $37^{\circ} \mathrm{C}$ ) disrupts the binding of $\mathrm{H}-\mathrm{NS}$ to DNA, resulting in the derepression of gene expression (Shahul Hameed et al., 2019) and this would explain the phenotypes observed in our study. Interestingly, the gene encoding the widely studied $\mathrm{H}-\mathrm{NS}$-like protein StpA exhibited higher expression levels at $42^{\circ} \mathrm{C}$ compared to $37^{\circ} \mathrm{C}$ which is in line with our findings (Muller et al., 2010). Surprisingly, we saw little difference in plasmid-mediated phenotypes between 25 and $37^{\circ} \mathrm{C}$, which suggest that regulatory pathways other than phns are important for the regulation of plasmid traits at $25^{\circ} \mathrm{C}$. Thus, our results proved that the variation of $\mathrm{H}$-NS expression contributes to the different phenotypes of plasmids at $30^{\circ} \mathrm{C}, 42^{\circ} \mathrm{C}$, and $37^{\circ} \mathrm{C}$.

The optimal function of IncX 3 plasmids at $37^{\circ} \mathrm{C}$ contrasts with that of IncHI1 plasmids like R27, which exhibit highest transfer frequencies at approximately $25^{\circ} \mathrm{C}$. In both cases, thermoregulation is exerted by plasmid-encoded $\mathrm{H}$-NS-like proteins. This suggests that different plasmid families have adapted to certain environmental conditions experienced by their hosts, and it will be interesting to determine whether transfer frequencies are temperature sensitive. It seems possible that the thermoregulation of plasmid functions influences the contributions of certain plasmid types to the dissemination of antibiotic resistance determinants in different environments. For example, IncHI1 plasmids might be expected to play a greater role in the transfer of resistance genes within environmental bacterial populations experiencing ambient temperatures of approximately $25^{\circ} \mathrm{C}$, while IncX 3 plasmids play a major role in the spread of resistance genes within mammalian enteric populations.

We acknowledge that our study has several limitations. First, our investigation focused on the plasmid-encoded gene phns to examine its modulation of plasmid characteristics under different temperatures. However, we could not take the interactions between host and plasmid regulators into consideration. For example, it has been reported that in E. coli chromosome-encoded temperature-sensor $\sigma-32$ is also involved in thermoregulation of plasmid conjugation (Rozwandowicz et al., 2019). Secondly, although we found that at 30 and $42^{\circ} \mathrm{C}$, IncX3 plasmid-bearing E. coli exhibits lower transfer ability and stability, various IncX3 plasmids have been isolated from natural environments (Cheng et al., 2019). Therefore, we speculate that IncX3 plasmid-bearing host might require compensatory evolution for long-term adaptation to new environments with temperatures that are different to the mammalian gut (Zwanzig et al., 2019). Further studies of plasmid-host co-evolution need to be conducted to explore the mechanism by which plasmids and hosts reach a fitness optimum at various temperature.

\section{CONCLUSION}

In conclusion, this report describes the differential impact of physiological and environmental temperature on IncX3 plasmid phenotypes. The IncX3 plasmid studied here exhibited a higher transfer frequency, was more stable and imposed a lower fitness cost at $37^{\circ} \mathrm{C}$ than at other temperatures. Temperature-regulated variation involve a plasmid-encoded H-NS-like protein, which acts as a thermosensor and is upregulated at 30 and $42^{\circ} \mathrm{C}$. The $\mathrm{H}$-NS-like protein inhibits conjugation genes and appears to decrease plasmid stability and host fitness. Our findings suggest that $b l a_{\mathrm{NDM}}$-bearing IncX3 plasmids have evolved to persist and transfer optimally in the mammalian gut, which will complicate the control of the spread of carbapenemresistant pathogens.

\section{DATA AVAILABILITY STATEMENT}

The original contributions presented in the study are included in the article/Supplementary Material, and further inquiries can be directed to the corresponding authors.

\section{AUTHOR CONTRIBUTIONS}

All authors listed have made a substantial, direct, and intellectual contribution to the work, and approved it for publication.

\section{FUNDING}

The research was supported by the National Natural Science Foundation of China (81772238), the Project of International Cooperation and Exchanges NSFC (8181101332), and the Guangzhou Municipal Science and Technology Bureau (project no. 201607020044) and the Medical Research Council (MR/ S013660/1).

\section{ACKNOWLEDGMENTS}

We acknowledge Prof. Yu Yunsong and Dr. Jiang Yan from Sir Run Shaw Hospital, Zhejiang University School of Medicine for kindly providing strain ATCC 700603. We thank Doctor Zhong LanLan in Sun-Yat University for kindly helping with conjugation experiments.

\section{SUPPLEMENTARY MATERIAL}

The Supplementary Material for this article can be found online at: https://www.frontiersin.org/articles/10.3389/fmicb.2021.765492/ full\#supplementary-material 


\section{REFERENCES}

Bush, K., and Bradford, P. A. (2020). Epidemiology of beta-lactamase-producing pathogens. Clin. Microbiol. Rev. 33:e0047-19. doi: 10.1128/CMR.00047-19

Chen, C.-L., Wang, C.-Y., Chu, C., Su, L.-H., and Chiu, C.-H. (2009). Functional and molecular characterization of pSE34 encoding a type IV secretion system in Salmonella enterica serotype Enteritidis phage type 34. FEMS Immunol. Med. Microbiol. 57, 274-283. doi: 10.1111/j.1574-695X.2009.00612.x

Cheng, P., Li, F., Liu, R., Yang, Y., Xiao, T., Ishfaq, M., et al. (2019). Prevalence and molecular epidemiology characteristics of carbapenem-resistant Escherichia coli in Heilongjiang Province, China. Infect. Drug Resist. 12, 2505-2518. doi: 10.2147/IDR.S208122

Dorman, C. J., and Ni Bhriain, N. (2020). CRISPR-Cas, DNA supercoiling, and nucleoid-associated proteins. Trends Microbiol. 28, 19-27. doi: 10.1016/j. tim.2019.08.004

Doyle, M., Fookes, M., Ivens, A., Mangan, M. W., Wain, J., and Dorman, C. J. (2007). An H-NS-like stealth protein aids horizontal DNA transmission in bacteria. Science 315, 251-252. doi: 10.1126/science.1137550

Forns, N., Banos, R. C., Balsalobre, C., Juarez, A., and Madrid, C. (2005). Temperature-dependent conjugative transfer of R27: role of chromosomeand plasmid-encoded Hha and H-NS proteins. J. Bacteriol. 187, 3950-3959. doi: 10.1128/JB.187.12.3950-3959.2005

Gao, H., Liu, Y., Wang, R., Wang, Q., Jin, L., and Wang, H. (2020). The transferability and evolution of NDM-1 and KPC-2 co-producing Klebsiella pneumoniae from clinical settings. EBioMedicine 51:102599. doi: 10.1016/j. ebiom.2019.102599

Ho, P. L., Cheung, Y. Y., Lo, W. U., Li, Z., Chow, K. H., Lin, C. H., et al. (2013). Molecular characterization of an atypical IncX3 plasmid pKPC-NY79 carrying bla KPC-2 in a Klebsiella pneumoniae. Curr. Microbiol. 67, 493-498. doi: $10.1007 /$ s00284-013-0398-2

Johnson, T. J., Singer, R. S., Isaacson, R. E., Danzeisen, J. L., Lang, K., Kobluk, K., et al. (2015). In vivo transmission of an IncA/C plasmid in Escherichia coli depends on tetracycline concentration, and acquisition of the plasmid results in a variable cost of fitness. Appl. Environ. Microbiol. 81, 3561-3570. doi: 10.1128/AEM.04193-14

Khan, A. U., Maryam, L., and Zarrilli, R. (2017). Structure, genetics and worldwide spread of New Delhi Metallo-beta-lactamase (NDM): a threat to public health. BMC Microbiol. 17:101. doi: 10.1186/s12866-017-1012-8

Liakopoulos, A., van der Goot, J., Bossers, A., Betts, J., Brouwer, M. S. M., Kant, A., et al. (2018). Genomic and functional characterisation of IncX3 plasmids encoding blaSHV-12 in Escherichia coli from human and animal origin. Sci. Rep. 8:7674. doi: 10.1038/s41598-018-26073-5

Liu, B., Shui, L., Zhou, K., Jiang, Y., Li, X., Guan, J., et al. (2020). Impact of plasmid-encoded H-NS-like protein on blaNDM-1-bearing IncX3 plasmid in Escherichia coli. J. Infect. Dis. 221, S229-S236. doi: 10.1093/infdis/jiz567

Logan, L. K., and Weinstein, R. A. (2017). The epidemiology of carbapenemresistant Enterobacteriaceae: the impact and evolution of a global menace. J. Infect. Dis. 215, S28-S36. doi: 10.1093/infdis/jiw282

Ma, T., Fu, J., Xie, N., Ma, S., Lei, L., Zhai, W., et al. (2020). Fitness cost of blaNDM-5-carrying p3R-IncX3 plasmids in wild-type NDM-free Enterobacteriaceae. Microorganisms 8:377. doi: 10.3390/microorganisms8030377

Matsumura, Y., Peirano, G., and Pitout, J. D. D. (2018). Complete genome sequence of Escherichia coli J53, an azide-resistant laboratory strain used for conjugation experiments. Genome Announc. 6:e00433-18. doi: 10.1128/ genomeA.00515-18

Muller, C. M., Schneider, G., Dobrindt, U., Emody, L., Hacker, J., and Uhlin, B. E. (2010). Differential effects and interactions of endogenous and horizontally acquired H-NS-like proteins in pathogenic Escherichia coli. Mol. Microbiol. 75, 280-293. doi: 10.1111/j.1365-2958.2009.06995.x

Nigg, A., Brilhante, M., Dazio, V., Clément, M., Collaud, A., Gobeli Brawand, S., et al. (2019). Shedding of OXA-181 carbapenemase-producing Escherichia coli from companion animals after hospitalisation in Switzerland: an outbreak in 2018. Euro Surveill. 24:1900071. doi: 10.2807/1560-7917.ES.2019.24.39. 1900071
Rose, R. E. (1988). The nucleotide sequence of pACYC184. Nucleic Acids Res. 16:355. doi: 10.1093/nar/16.14.7191

Rozwandowicz, M., Brouwer, M. S. M., Mughini-Gras, L., Wagenaar, J. A., Gonzalez-Zorn, B., Mevius, D. J., et al. (2019). Successful host adaptation of IncK2 plasmids. Front. Microbiol. 10:2384. doi: 10.3389/fmicb.2019.02384

Shahul Hameed, U. F., Liao, C., Radhakrishnan, A. K., Huser, F., Aljedani, S. S., Zhao, X., et al. (2019). H-NS uses an autoinhibitory conformational switch for environment-controlled gene silencing. Nucleic Acids Res. 47, 2666-2680. doi: 10.1093/nar/gky1299

Shintani, M., Suzuki-Minakuchi, C., and Nojiri, H. (2015). Nucleoid-associated proteins encoded on plasmids: occurrence and mode of function. Plasmid 80, 32-44. doi: 10.1016/j.plasmid.2015.04.008

Takeda, T., Yun, C. S., Shintani, M., Yamane, H., and Nojiri, H. (2011). Distribution of genes encoding nucleoid-associated protein homologs in plasmids. Int. J. Evol. Biol. 2011:685015. doi: 10.4061/2011/685015

Wang, Y., Tian, G. B., Zhang, R., Shen, Y., Tyrrell, J. M., Huang, X., et al. (2017). Prevalence, risk factors, outcomes, and molecular epidemiology of mcr-1-positive Enterobacteriaceae in patients and healthy adults from China: an epidemiological and clinical study. Lancet Infect. Dis. 17, 390-399. doi: 10.1016/S1473-3099(16)30527-8

Wang, Y., Tong, M.-K., Chow, K.-H., Cheng, V. C.-C., Tse, C. W.-S., Wu, A. K.L., et al. (2018). Occurrence of highly conjugative IncX3 epidemic plasmid carrying blaNDM in Enterobacteriaceae isolates in geographically widespread areas. Front. Microbiol. 9:2272. doi: 10.3389/fmicb.2018.03354

Wein, T., Hulter, N. F., Mizrahi, I., and Dagan, T. (2019). Emergence of plasmid stability under non-selective conditions maintains antibiotic resistance. Nat. Commun. 10:2595. doi: 10.1038/s41467-019-10600-7

Zhai, R., Fu, B., Shi, X., Sun, C., Liu, Z., Wang, S., et al. (2020). Contaminated in-house environment contributes to the persistence and transmission of NDM-producing bacteria in a Chinese poultry farm. Environ. Int. 139:105715. doi: 10.1016/j.envint.2020.105715

Zhang, Q., Lv, L., Huang, X., Huang, Y., Zhuang, Z., Lu, J., et al. (2019). Rapid increase in carbapenemase-producing Enterobacteriaceae in retail meat driven by the spread of the bla (NDM-5)-carrying IncX3 plasmid in China from 2016 to 2018. Antimicrob. Agents Chemother. 63:e00573-19. doi: 10.1128/ AAC.00573-19

Zhang, Y., Wang, Q., Yin, Y., Chen, H., Jin, L., Gu, B., et al. (2018). Epidemiology of carbapenem-resistant Enterobacteriaceae infections: report from the China CRE network. Antimicrob. Agents Chemother. 62:e01882-17. doi: 10.1128/ AAC.01882-17

Zhou, H., Zhang, K., Chen, W., Chen, J., Zheng, J., Liu, C., et al. (2020). Epidemiological characteristics of carbapenem-resistant Enterobacteriaceae collected from 17 hospitals in Nanjing district of China. Antimicrob. Agents Chemother. 9:15. doi: 10.1186/s13756-019-0674-4

Zwanzig, M., Harrison, E., Brockhurst, M. A., Hall, J. P. J., Berendonk, T. U., and Berger, U. (2019). Mobile compensatory mutations promote plasmid survival. mSystems 4:e00186-18. doi: 10.1128/mSystems.00186-18

Conflict of Interest: The authors declare that the research was conducted in the absence of any commercial or financial relationships that could be construed as a potential conflict of interest.

Publisher's Note: All claims expressed in this article are solely those of the authors and do not necessarily represent those of their affiliated organizations, or those of the publisher, the editors and the reviewers. Any product that may be evaluated in this article, or claim that may be made by its manufacturer, is not guaranteed or endorsed by the publisher.

Copyright (๑) 2022 Baomo, Lili, Moran, van Schaik and Chao. This is an openaccess article distributed under the terms of the Creative Commons Attribution License (CC BY). The use, distribution or reproduction in other forums is permitted, provided the original author(s) and the copyright owner(s) are credited and that the original publication in this journal is cited, in accordance with accepted academic practice. No use, distribution or reproduction is permitted which does not comply with these terms. 